\title{
DESCRIÇÃO DE SINTOMAS VISUAIS EM FUNÇÃO DAS DEFICIÊNCIAS DE MACRONUTRIENTES EM MUDAS DE CEREJEIRA (Amburana acreana)
}

\author{
Cristiane Ramos Vieira ${ }^{1}$, Oscarlina Lúcia dos Santos Weber² ${ }^{2}$ José Fernando Scaramuzza ${ }^{3}$, \\ Anne Caroline Costa ${ }^{4}$, Thaianny Rodrigues de Souza ${ }^{4}$
}

\begin{abstract}
${ }^{1}$ Eng $^{\mathrm{a}}$. Florestal, M.Sc., Doutoranda em Agricultura Tropical, UFMT, Cuiabá, Mato Grosso, Brasil - cris00986@ hotmail.com ${ }^{2}$ Eng . Agrônoma e Química, Dr ${ }^{\mathrm{a}}$., Depto. de Solos e Engenharia Rural, UFMT, Cuiabá, Mato Grosso, Brasil - oscsan@uol.com.br ${ }^{3}$ Eng. Agrônomo, Dr., Depto. de Solos e Engenharia Rural, UFMT, Cuiabá, Mato Grosso, Brasil - jscaramuzza@uol.com.br ${ }^{4}$ Acadêmica de Eng. Florestal, UFMT, Cuiabá, Mato Grosso, Brasil - anne_ccosta@hotmail.com; thaiannyrodrigues@hotmail.com
\end{abstract}

Recebido para publicação: 28/10/2010 - Aceito para publicação: 26/07/2011

\begin{abstract}
Resumo
Este trabalho teve por objetivo identificar a sintomatologia visual das carências de macronutrientes e produção de massa seca em mudas de cerejeira (Amburana acreana), cultivadas em solução nutritiva. As mudas de cerejeira foram produzidas em tubetes de $180 \mathrm{~cm}^{3} \mathrm{e}$ posteriormente transplantadas para sacolas plásticas $(17 \times 10 \mathrm{~cm}$ ). Ao atingirem $20 \mathrm{~cm}$ de altura, foram transplantadas para tubos de PVC preenchidos com areia e submetidas por 30 dias a solução nutritiva completa, correspondente ao período de adaptação. Em seguida, as mudas foram submetidas por 90 dias a soluções nutritivas completa e com omissão de $\mathrm{N}, \mathrm{P}, \mathrm{K}, \mathrm{Ca}, \mathrm{Mg}, \mathrm{S}$, constituindo sete tratamentos e três repetições em delineamento inteiramente casualizado. A cada 30 dias, as mudas foram avaliadas em altura e diâmetro de colo e os sintomas de deficiência foram identificados. Após os 90 dias de aplicação de solução nutritiva, as mudas foram secas em estufa e a biomassa seca foi determinada. Concluiu-se que $\mathrm{P}, \mathrm{S}$ e $\mathrm{N}$ foram os macronutrientes que mais limitaram o crescimento das mudas de Amburana acreana e $\mathrm{P}$ e $\mathrm{S}$, a produção de matéria seca. Houve maior absorção de N, P e K na parte aérea das mudas de cerejeira e de $\mathrm{Ne} \mathrm{Mg}$ pelo sistema radicular.

Palavras-chave: Espécie florestal; viveiro florestal; nutrição de plantas.
\end{abstract}

\begin{abstract}
Description of visual symptoms under macronutrients deficiency in seedlings of cerejeira (Amburana acreana ducke). This study aimed to identify the visual symptoms of deficiencies of nutrients and dry mass production in seedlings of cherry (Amburana acreana), grown in nutrient solution. Cherry seedlings were grown in tubes $180 \mathrm{~cm}^{3}$ and later transplanted into plastic bags $(17 \times 10 \mathrm{~cm})$. Upon reaching $20 \mathrm{~cm}$ height were transplanted to PVC tubes filled with sand and subjected to 30 days to complete nutritive solution, corresponding to the period of adaptation. Then the seedlings were subjected for 90 days, to complete nutrient solutions and with the omission of $\mathrm{N}, \mathrm{P}, \mathrm{K}, \mathrm{Ca}, \mathrm{Mg}, \mathrm{S}$, constituting seven treatments and three replications in a randomized design. Every 30 days, the seedlings were assessed for height and diameter, and deficiency symptoms were identified and photographed. After 90 days of application of nutrient solution, the seedlings were dried and the dry biomass was determinate. It was concluded that $\mathrm{P}, \mathrm{S}$ and $\mathrm{N}$ were the macronutrients that limit the growth of seedlings Amburana acreana and $\mathrm{P}$ and $\mathrm{S}$, the dry mass production. There was greater absorption of $\mathrm{N}, \mathrm{P}$ and $\mathrm{K}$ in shoots of the seedlings and $\mathrm{N}$ and $\mathrm{Mg}$ by root.

Keywords: Amazonian species; nursery; plant nutrition.
\end{abstract}

\section{INTRODUÇÃO}

Quase $50 \%$ das florestas tropicais úmidas naturais existentes estão na América tropical e, desse total, mais de $80 \%$ (50\% no Brasil $+30 \%$ em outros países da América do Sul) se encontram na região amazônica (HUMMEL; MINETTE, 1990).

Devido à exploração não sustentável e/ou à diminuição da cobertura florestal, consequência do desmatamento recorrente nessa região, muitas das espécies locais se encontram na lista das vulneráveis à 
extinção. Sendo assim, estudos das espécies nativas amazônicas têm se intensificado, já que muitas delas possuem grande potencial econômico, porém ainda não há informações suficientes que levem ao conhecimento correto a seu respeito, bem como à sua exploração de forma menos impactante para o ambiente.

O desconhecimento sobre a ecologia das espécies nativas restringe sua utilização e ameaça sua conservação, uma vez que a velocidade da degradação ambiental tem sido superior aos esforços para garantir a manutenção da biodiversidade. Há risco de perda de recursos genéticos insubstituíveis, já que muitas dessas espécies podem possuir propriedades medicinais desconhecidas, além de apresentarem grande interesse econômico. Trata-se de recursos de grande potencial que necessitam ser explorados de forma sustentável e racional, para que se garanta a sua continuidade e sua exploração pelas gerações futuras (BELLO et al., 2008). Nesse âmbito, ressalte-se também a carência de informações acerca das exigências nutricionais dessas espécies.

Entre as espécies com potencial econômico encontra-se a Amburana acreana (Ducke) A. C. Sm., da família Fabaceae, conhecida vulgarmente como cerejeira. Essa espécie apresenta forma arborescente de alto fuste, ocorrendo em matas altas e fechadas na Amazônia ocidental do Brasil (AM, AC, RO e MT) e da Bolívia (CARVALHO, 1994).

Cada espécie pode ter características genéticas individuais, e para que sua utilização (ou até produção) possa ser a mais correta possível, é necessário que se conheçam as exigências nutricionais de cada uma.

Dentro dessa linha do conhecimento, Fontes (2001) recomenda que, para se fazer o diagnóstico do estado nutricional da planta, é fundamental interpretar os resultados da análise foliar, que consiste na comparação dos valores da concentração dos nutrientes na amostra-problema com os valores padrões publicados em tabelas ou verificados em plantas normais, decidindo se há ou não deficiência do elemento analisado. Como as folhas são utilizadas para análise foliar, por terem maior atividade metabólica, o diagnóstico da deficiência, segundo Malavolta (1992), é facilitado. Para tal, a diagnose foliar na fase de viveiro deve levar em consideração o estádio de desenvolvimento das mudas, pois a concentração dos nutrientes nas folhas diminui com a idade das mudas (SILVEIRA et al., 2001).

O conhecimento dos sintomas é importante tanto na fase de viveiro quanto na de campo, para decidir se haverá adubação, qual tipo de adubo utilizar e qual quantidade. De acordo com Fontes (2001), os sintomas observados nas plantas-problema precisam ser descritos apropriadamente. Ao descrevê-los, é necessário atenção ao início do aparecimento dos sintomas (ou sintomas primários), para a exclusão daqueles causados por fatores bióticos e abióticos.

$\mathrm{O}$ crescimento das espécies florestais pode ser limitado caso encontrem restrições nutricionais, pois este se relaciona com a massa seca e com os nutrientes absorvidos pela planta. Portanto, se essas espécies forem adubadas com as quantidades corretas de fertilizante, podem atingir o máximo de crescimento.

Como são poucos os estudos das necessidades nutricionais de espécies florestais, este estudo teve por objetivo descrever a sintomatologia das deficiências de macronutrientes e verificar quais os macronutrientes mais limitantes ao crescimento de mudas de Amburana acreana (cerejeira) cultivadas em solução nutritiva.

\section{MATERIAL E MÉTODOS}

A produção das mudas foi realizada no viveiro florestal da Faculdade de Engenharia Florestal da Universidade Federal de Mato Grosso, com sementes coletadas de árvores existentes no campus da Universidade e semeadas em tubetes de polipropileno com capacidade para $180 \mathrm{~cm}^{3}$, contendo combinação de vermiculita, Plantmax ${ }^{\circledR}$ e torta de filtro.

O experimento foi conduzido em casa de vegetação da Faculdade de Agronomia e Medicina Veterinária (FAMEV) da Universidade Federal de Mato Grosso, Cuiabá, MT, situada nas coordenadas de $15^{\circ} 36^{\prime}$ S e $56^{\circ} 04^{\prime} \mathrm{W}$, altitude de $200 \mathrm{~m}$. Nessa região, o clima predominante, segundo classificação de Köppen, é o tropical de savana.

O delineamento experimental foi o inteiramente casualizado com sete tratamentos: completo, -N, $-\mathrm{P},-\mathrm{K},-\mathrm{Ca},-\mathrm{Mg}$ e $-\mathrm{S}$ e três repetições, sendo cada parcela constituída por uma planta. Utilizaram-se soluções preparadas conforme Sarruge (1975). Primeiramente, ao atingir cerca de $20 \mathrm{~cm}$ de altura, as mudas foram transplantadas para tubos de PVC de $40 \mathrm{~cm}$ de comprimento e $10 \mathrm{~cm}$ de diâmetro, preenchidos com areia previamente lavada e tratada com hipoclorito, e submetidas, durante 30 dias, à aplicação de $50 \mathrm{~mL}$ de solução nutritiva completa, renovada a cada cinco dias. Nessa etapa, as mudas 
foram irrigadas diariamente, exceto no dia de aplicação da solução completa. Após o período de adaptação, as mudas foram submetidas, durante 90 dias, à aplicação de $50 \mathrm{~mL}$ de soluções completa e com omissões de macronutrientes, reaplicadas a cada cinco dias.

Os sintomas de deficiências foram verificados e descritos a cada 15 dias. Além disso, a cada 30 dias foi realizada a medição das mudas, sendo verificados: altura da parte aérea $(\mathrm{H})$, em $\mathrm{cm}$, com régua graduada, efetuando a medição de toda a parte acima do substrato; diâmetro de coleto (DC), em mm, com paquímetro digital de $150 \mathrm{~mm}$ da marca PROFIELD®, efetuando medição na região da planta rente ao substrato; e biomassa seca das partes aérea (MSPA) e radicular (MSPR), verificados ao final do experimento.

Após 90 dias, as mudas foram retiradas dos recipientes, lavadas com água destilada, seccionadas em parte aérea e radicular, colocadas em sacos de papel e secas em estufa de circulação de ar forçado a $65^{\circ} \mathrm{C}$ até atingir peso constante. $\mathrm{O}$ material seco foi pesado em balança analítica com precisão de $0,05 \mathrm{~g}$ e, após pesagem, foi moído em moinho do tipo Wiley e acondicionado em sacolas plásticas.

As amostras moídas foram submetidas à digestão nitro-perclórica e sulfúrica, conforme Malavolta et al. (1997), e posteriormente determinaram-se os teores de N por semimicro Kjeldahl; P por colorimetria do metavanadato; S por turbidimetria do sulfato de bário; K por fotometria de chama de emissão; $\mathrm{Ca}$ e $\mathrm{Mg}$ por quelatometria com EDTA, sendo que os teores dos mesmos foram obtidos por diferença.

Os resultados foram submetidos à análise de variância e comparação das médias pelo teste de Tukey a 5\% de probabilidade, utilizando-se o Assistat 7.5 beta.

\section{RESULTADOS E DISCUSSÃO}

\section{Biometria e produção de biomassa seca da partes aérea e raízes}

As medidas da altura, do diâmetro de colo e da biomassa seca nas partes aérea e radicular de mudas de Amburana acreana submetidas às soluções nutritivas durante três meses estão na tabela 1 . As avaliações mensais de altura e diâmetro de colo não foram significativas durante todo o experimento, possivelmente devido à produção das mudas ter ocorrido em substrato orgânico, que proporcionou crescimento em altura e em diâmetro às plantas mesmo com omissão dos macronutrientes. Além disso, as mudas passaram por um período de adaptação.

Tabela 1. Altura, diâmetro de colo e produção de biomassa na parte aérea e raízes de mudas de Amburana acreana em soluções com omissão de macronutrientes.

Table 1. Height, diameter and dry biomass production in the aerial part and roots of Amburana acreana in solutions with the omission of macronutrients.

\begin{tabular}{lcccccccc}
\hline \multirow{2}{*}{ Soluções } & H1 & H2 & H3 & DC1 & DC2 & DC3 & MSPA (g) & MSPR (g) \\
\cline { 2 - 8 } & $34,00 \mathrm{a}$ & $35,00 \mathrm{a}$ & $41,67 \mathrm{a}$ & $2,40 \mathrm{a}$ & $2,91 \mathrm{a}$ & $3,13 \mathrm{a}$ & $2,46 \mathrm{ab}$ & $1,79 \mathrm{ab}$ \\
Completa & $30,67 \mathrm{a}$ & $35,33 \mathrm{a}$ & $35,67 \mathrm{a}$ & $2,49 \mathrm{a}$ & $3,02 \mathrm{a}$ & $3,29 \mathrm{a}$ & $1,71 \mathrm{bcd}$ & $1,89 \mathrm{ab}$ \\
$-\mathrm{N}$ & $25,33 \mathrm{a}$ & $26,33 \mathrm{a}$ & $29,33 \mathrm{a}$ & $1,79 \mathrm{a}$ & $2,27 \mathrm{a}$ & $2,34 \mathrm{a}$ & $0,83 \mathrm{~d}$ & $0,17 \mathrm{c}$ \\
$-\mathrm{P}$ & $32,33 \mathrm{a}$ & $34,67 \mathrm{a}$ & $38,33 \mathrm{a}$ & $2,65 \mathrm{a}$ & $2,94 \mathrm{a}$ & $3,06 \mathrm{a}$ & $2,25 \mathrm{abc}$ & $1,43 \mathrm{~b}$ \\
$-\mathrm{K}$ & $34,33 \mathrm{a}$ & $38,33 \mathrm{a}$ & $43,00 \mathrm{a}$ & $3,06 \mathrm{a}$ & $3,33 \mathrm{a}$ & $3,39 \mathrm{a}$ & $2,52 \mathrm{ab}$ & $2,65 \mathrm{a}$ \\
$-\mathrm{Ca}$ & $36,33 \mathrm{a}$ & $39,00 \mathrm{a}$ & $44,33 \mathrm{a}$ & $2,41 \mathrm{a}$ & $2,79 \mathrm{a}$ & $3,10 \mathrm{a}$ & $3,20 \mathrm{a}$ & $1,97 \mathrm{ab}$ \\
$-\mathrm{Mg}$ & $27,00 \mathrm{a}$ & $29,67 \mathrm{a}$ & $32,67 \mathrm{a}$ & $1,83 \mathrm{a}$ & $2,24 \mathrm{a}$ & $2,55 \mathrm{a}$ & $1,27 \mathrm{~cd}$ & $0,37 \mathrm{c}$ \\
-S & 27,47 & 24,74 & 25,08 & 25,97 & 27,93 & 25,02 & 18,45 & 24,27 \\
\hline CV (\%) & $1.335,14$ & $1.366,95$ & $1.824,57$ & 8,92 & 11,34 & 10,50 & 45,43 & 67,17 \\
\hline D-padrão & &
\end{tabular}

Médias seguidas de mesma letra (em coluna) não diferem entre si pelo teste Tukey a 5\%. H1, H2 e H3: altura da parte aérea da muda após um mês, dois meses e três meses, respectivamente, de aplicação das soluções nutritivas; DC1, DC2 e DC3: diâmetro de colo após um, dois e três meses, respectivamente, de aplicação das soluções nutritivas; MSPA: biomassa seca da parte aérea; MSPR: biomassa seca da parte radicular; IQD: índice de qualidade de Dickson; D-padrão: desvio padrão das variáveis mensuradas.

Contrariamente ao que ocorreu no crescimento em altura e diâmetro, na produção de biomassa houve significância. Observa-se que as mudas no tratamento completo não obtiveram as maiores produções.

Não houve redução na produção de biomassa na parte aérea das mudas de cerejeira submetidas à omissão de Mg. Na parte radicular, essa omissão teve valor igual ao do tratamento completo. 
A omissão de $\mathrm{P}$ foi a que mais limitou a produção de biomassa na parte aérea e uma das que mais reduziram a produção na parte radicular. Malavolta et al. (1997) mencionam que um dos efeitos mais marcantes da deficiência de P é a acentuada redução no crescimento como um todo. Portanto, as omissões de macronutrientes não limitaram o crescimento em altura e diâmetro, mas foram determinantes para a redução no crescimento em massa.

As omissões de $\mathrm{S}$ e de $\mathrm{N}$ também limitaram a produção de biomassa na parte aérea. No caso de $\mathrm{N}$, a redução foi de $30,49 \%$, e de $51,63 \%$ no caso do $\mathrm{S}$, em comparação com o tratamento completo. O tratamento -S e o -P foram os que mais reduziram a biomassa na parte radicular.

A omissão de $\mathrm{K}$ também reduziu a produção de biomassa na parte radicular, nesse caso, a produção foi superior apenas aos tratamentos -P e -S. Lima (2002) observou redução no número e tamanho das folhas, assim como no porte da planta de cupuaçuzeiro quando da ausência de $S$.

$\mathrm{Na}$ omissão de $\mathrm{Ca}$, a produção de biomassa na parte aérea foi significativamente igual à do tratamento completo. Na parte radicular, a ausência de Ca não limitou a produção de biomassa, obtendo média superior à do tratamento completo. Kaul et al. (1968), Sarcinelli et al. (2004) e Wallau et al. (2008) também observaram que a omissão de Ca na solução não reduziu a produção de biomassa das plantas estudadas, Eucalyptus, Acacia holosericea e Swietenia macrophylla, respectivamente.

$\mathrm{Na}$ parte radicular, a produção de biomassa nos tratamentos $-\mathrm{Mg}$ e $-\mathrm{N}$ foi significativamente igual à do tratamento completo.

Portanto, com relação à biomassa seca, observaram-se as menores produções ao se omitirem $\mathrm{P}$, $\mathrm{K}$ e $\mathrm{S}$, na parte aérea, sendo limitada na seguinte ordem: $\mathrm{P}>\mathrm{S}>\mathrm{N}>\mathrm{K}>\mathrm{Completa}>\mathrm{Ca}>\mathrm{Mg}$. Na parte radicular, as menores produções foram observadas ao se omitirem P, S e K, limitada na seguinte ordem: $\mathrm{P}>\mathrm{S}>\mathrm{K}>$ Completa $>\mathrm{N}>\mathrm{Mg}>\mathrm{Ca}$.

\section{Sintomatologia visual das deficiências de macronutrientes}

Nitrogênio: Após 30 dias de aplicação da solução com omissão de N, observaram-se mudas com folhas de coloração verde claro ou amarelada, passando para um amarelecimento total das folhas novas com posterior perda total de cor, ficando totalmente esbranquiçadas, com secamento e queda de folhas. Essa observação também foi feita por Ramos (2006), cujas folhas apresentaram-se, inicialmente com uma coloração verde-amarelada, com um pequeno gradiente de intensidade nas mais velhas. Segundo Malézieux e Bartholomew (2003), quando o N está deficiente, as folhas são verde-amareladas a amarelas. Isso, segundo Simões e Couto (1973), acontece porque o N está presente em vários compostos que são de grande importância fisiológica, como a produção de clorofila. Além da coloração, observaram-se enrugamento nas bordas das folhas e uma leva tortuosidade do caule.

Fósforo: As folhas das mudas de cerejeira ficaram com uma coloração verde mais claro, que progrediu para amarelecimento bem acentuado e, posteriormente, para uma total perda de coloração das folhas. Sintomas parecidos com os observados por Ramos (2006), em que as plantas sob deficiência de fósforo apresentaram, no começo, amarelecimento nas folhas mais novas. Sintoma semelhantes aos observados por Barroso et al. (2005), em mudas de teca. Além disso, observou-se também enrugamento das bordas foliares.

Potássio: As folhas apresentaram pontos de coloração verde mais claro, progredindo para acentuada perda de cor, ficando somente com a região da nervura central com coloração esverdeada. Essas folhas, após cerca de 30 dias, começaram a enrugar, ficando com as bordas com aspecto retorcido. Além disso, algumas folhas novas atrofiaram, o que prejudicou seu desenvolvimento. Mendonça et al. (1999) também observaram que a omissão de K causou enrugamento das folhas de aroeira, que posteriormente apresentaram aspecto queimado. Em Wallau et al. (2008), as folhas velhas se caracterizaram por um encarquilhamento para baixo.

Cálcio: As folhas apresentaram, inicialmente, manchas de coloração verde claro, porém, com a coloração normal na região das nervuras. Esse sintoma progrediu e, após 90 dias, as folhas ficaram praticamente sem coloração, porém com um tom esverdeado na região da nervura central, sendo que algumas dessas folhas ficaram com aspecto retorcido ou, ainda, com começo de necrose nas bordas. Sintomas condizentes com os descritos por Silveira et al. (2002), em que as folhas novas apresentaram-se deformadas e retorcidas, assim como observado por Barroso et al. (2005), Sarcinelli et al. (2004) e Marques et al. (2004). 
Magnésio: Após 30 dias, as folhas começaram a apresentar manchas de coloração verdeamarelada. Sintoma condizente com Ramos (2006), que menciona que plantas deficientes de $\mathrm{Mg}$ apresentam uma coloração verde-amarelada nas folhas. No presente caso, esse sintoma progrediu para um acentuado amarelecimento que começou pelas bordas das folhas, fazendo com que posteriormente ficassem totalmente sem coloração, apresentando ainda bordas com aspecto ondulado.

Enxofre: Os sintomas nesse tratamento começaram após 30 dias de aplicação da solução nutritiva, quando folhas começaram a apresentar uma coloração um pouco mais clara que as folhas de plantas sadias e, após 90 dias, perderam totalmente a coloração. Em estudo realizado por Locatelli et al. (2006), observou-se que os sintomas de deficiência de $\mathrm{S}$ iniciaram com um verde-claro, passando a verde-amarelo e progredindo para amarelo intenso. Além desses sintomas, pôde-se verificar que as mudas apresentaram crescimento apical afetado por conta do atrofiamento do ápice, sendo que algumas folhas velhas também começaram a apresentar atrofiamento das bordas.

\section{Teores de macronutrientes}

Os resultados dos teores de macronutrientes em mudas de cerejeira, nas partes aérea e radicular encontram-se na tabela 2. Pode-se observar que o tratamento completo propiciou maior teor de $\mathrm{Ca}$ na parte aérea e de $\mathrm{Ca}$ e $\mathrm{S}$ no sistema radicular, não ocorrendo o mesmo com os demais nutrientes.

Tabela 2. Teores de macronutrientes em $\mathrm{g} \cdot \mathrm{kg}^{-1}$ nas partes aérea e radicular de mudas de Amburana acreana submetidas à omissão de macronutrientes.

Table 2. Contents of macronutrients in $\mathrm{g}^{\mathrm{kg}} \mathrm{g}^{-1}$, in aerial and root parts of seedlings of Amburana acreana subjected to omission of nutrients.

\begin{tabular}{|c|c|c|c|c|c|c|}
\hline Tratamento & $\mathbf{N}$ & $\mathbf{P}$ & $\mathbf{K}$ & $\mathbf{C a}$ & Mg & $\mathbf{S}$ \\
\hline \multicolumn{7}{|c|}{ Parte aérea } \\
\hline Completo & $15,59 \mathrm{c}$ & $6,45 \mathrm{ab}$ & $7,00 \mathrm{~b}$ & $4,78 \mathrm{a}$ & $3,00 \mathrm{~b}$ & $0,44 \mathrm{bc}$ \\
\hline$-\mathrm{N}$ & $11,29 \mathrm{~d}$ & $5,78 \mathrm{bc}$ & $6,50 \mathrm{c}$ & $4,70 \mathrm{ab}$ & $3,50 \mathrm{ab}$ & $0,48 \mathrm{~b}$ \\
\hline$-\mathrm{P}$ & $21,93 \mathrm{a}$ & $5,74 \mathrm{c}$ & $7,83 \mathrm{a}$ & $4,43 a b c$ & $4,00 \mathrm{ab}$ & $0,86 \mathrm{a}$ \\
\hline$-K$ & $17,83 \mathrm{bc}$ & $4,65 \mathrm{~d}$ & $6,50 \mathrm{c}$ & $4,37 \mathrm{abc}$ & $6,50 \mathrm{a}$ & $0,25 \mathrm{~d}$ \\
\hline$-\mathrm{Ca}$ & $9,89 \mathrm{~d}$ & $6,98 \mathrm{a}$ & $6,50 \mathrm{c}$ & $4,37 \mathrm{abc}$ & $4,00 \mathrm{ab}$ & $0,28 \mathrm{~cd}$ \\
\hline$-\mathrm{Mg}$ & $15,49 \mathrm{c}$ & $5,35 \mathrm{c}$ & $6,33 \mathrm{c}$ & $4,23 \mathrm{bc}$ & $3,00 \mathrm{~b}$ & $0,78 \mathrm{a}$ \\
\hline$-S$ & $21,19 \mathrm{ab}$ & $5,42 \mathrm{c}$ & $7,50 \mathrm{a}$ & $4,17 \mathrm{c}$ & $4,50 \mathrm{ab}$ & $0,40 \mathrm{bcd}$ \\
\hline CV (\%) & 7,78 & 4,28 & 2,24 & 4,00 & 28,99 & 13,23 \\
\hline Desvio padrão & 397,47 & 11,26 & 6,45 & 1,35 & 45,64 & 1,06 \\
\hline \multicolumn{7}{|c|}{ Parte radicular } \\
\hline Completo & $8,49 \mathrm{~b}$ & $4,35 \mathrm{ab}$ & $4,67 \mathrm{~b}$ & $4,73 \mathrm{a}$ & $7,00 \mathrm{ab}$ & $1,54 \mathrm{a}$ \\
\hline$-\mathrm{N}$ & $5,41 \mathrm{~b}$ & $4,15 \mathrm{~b}$ & $4,33 \mathrm{~b}$ & $4,10 \mathrm{bc}$ & $11,50 \mathrm{a}$ & $0,44 \mathrm{~b}$ \\
\hline$-P$ & $14,84 \mathrm{a}$ & $4,45 \mathrm{ab}$ & $5,67 \mathrm{a}$ & $4,40 \mathrm{ab}$ & $3,75 \mathrm{~b}$ & $0,10 \mathrm{~b}$ \\
\hline$-\mathrm{K}$ & $7,93 \mathrm{~b}$ & $4,12 \mathrm{~b}$ & $4,33 \mathrm{~b}$ & $4,47 \mathrm{ab}$ & $6,50 \mathrm{~b}$ & $0,42 \mathrm{~b}$ \\
\hline$-\mathrm{Ca}$ & $5,23 \mathrm{~b}$ & $4,77 \mathrm{a}$ & $4,33 \mathrm{~b}$ & $4,40 \mathrm{ab}$ & $6,50 \mathrm{~b}$ & $0,27 \mathrm{~b}$ \\
\hline$-\mathrm{Mg}$ & $8,59 \mathrm{~b}$ & $4,60 \mathrm{a}$ & $4,33 \mathrm{~b}$ & $3,87 \mathrm{c}$ & $7,00 \mathrm{ab}$ & $0,49 \mathrm{~b}$ \\
\hline$-S$ & $9,33 \mathrm{~b}$ & $4,75 \mathrm{a}$ & $4,83 \mathrm{ab}$ & $4,40 \mathrm{ab}$ & $4,00 \mathrm{~b}$ & $0,45 \mathrm{~b}$ \\
\hline $\mathrm{CV}(\%)$ & 23 & 3,61 & 7,43 & 3,47 & 27,47 & 28,17 \\
\hline Desvio padrão & 238,43 & 1,66 & 6,07 & 1,69 & 163,82 & 4,20 \\
\hline
\end{tabular}

Nitrogênio: As maiores concentrações de $\mathrm{N}$ foram encontradas na parte aérea das mudas de cerejeira, o que ocorre por causa da fácil mobilidade desse nutriente. Na parte aérea, ao comparar com o tratamento completo, as omissões de $\mathrm{P}, \mathrm{K}$ e $\mathrm{S}$ causaram aumento na concentração de $\mathrm{N}$ nas plantas. No entanto, a maior redução na concentração de $\mathrm{N}$ foi observada quando ele foi subtraído. Dell et al. (1995) sugeriram para $E$. grandis x E. urophylla concentrações foliares de $\mathrm{N}$ numa faixa de 18 a $29 \mathrm{~g}^{\mathrm{kg}}{ }^{-1}$. Dessa forma, no presente caso, somente os tratamentos com omissão de $\mathrm{P}$ e de $\mathrm{S}$ promoveram condições de absorção de $\mathrm{N}$ em níveis favoráveis para a planta. Na parte radicular, a omissão de $\mathrm{P}$ provocou aumento na concentração de N. Nos outros tratamentos, a concentração de $\mathrm{N}$ foi considerada igual. Veloso et al. 
(1998) verificaram que os maiores teores de $\mathrm{N}$ nas folhas, caule e raízes foram observados com a omissão de P, K, Ca e S.

Fósforo: As maiores concentrações de $\mathrm{P}$ foram encontradas na parte aérea. A omissão de $\mathrm{Ca}$ aumentou, na parte aérea, a concentração de $\mathrm{P}$, quando comparado com o tratamento completo, como também ocorrido em Sgarbi et al. (1999). As omissões de P, Mg e S provocaram redução na concentração de P, porém essa redução foi ainda mais significativa ao se omitir K. Maynard (1970) observou aumento nos teores de $\mathrm{P}$ em plantas deficientes em K. Mendonça et al. (1999) também observaram o aumento nos teores de $\mathrm{P}$ nas folhas das mudas de aroeira do sertão com a omissão de S. Dell et al. (1995) sugeriram para $E$. grandis x E. urophylla concentração de $\mathrm{P}$ numa faixa de 2 a $6 \mathrm{gghg}^{-1}$. Dessa forma, neste experimento, os tratamentos completo e com omissão de Ca apresentaram concentrações acima do ideal. Na parte radicular, as omissões de $\mathrm{Ca}, \mathrm{Mg}$ e S provocaram aumento da concentração de $\mathrm{P}$ nas plantas, quando comparado com o tratamento completo. Já nas omissões de $\mathrm{N}$ e K, observou-se redução do teor de P.

Potássio: As menores concentrações de $\mathrm{K}$ foram encontradas na parte radicular das mudas de cerejeira. As omissões de $\mathrm{P}$ e S provocaram aumento no teor de $\mathrm{K}$, quando comparado com o tratamento completo, tanto na parte aérea quanto na radicular. Na parte radicular, ao se omitir K, a concentração desse nutriente foi igual à do tratamento completo. Na parte aérea, as omissões de $\mathrm{N}, \mathrm{K}, \mathrm{Ca}$ e $\mathrm{Mg}$ reduziram a concentração de K.

Cálcio: Maiores concentrações de Ca foram observadas em mudas do tratamento completo, tanto na parte aérea quanto na radicular. Na parte aérea, as omissões de $\mathrm{Mg}$ e $\mathrm{S}$ provocaram as maiores reduções na concentração de $\mathrm{Ca}$. Na parte radicular, nas omissões de $\mathrm{P}, \mathrm{K}, \mathrm{Ca}$ e $\mathrm{S}$, a concentração de Ca foi considerada igual. Porém, nas omissões de $\mathrm{N}$ e $\mathrm{Mg}$, observou-se redução. A concentração de $\mathrm{Ca}$, de acordo com Dell et al. (1995), deve estar numa faixa de 2,1 a 7,5 g. $\mathrm{kg}^{-1}$, portanto todos os tratamentos testados neste experimento apresentaram concentrações de Ca dentro da faixa ideal.

Magnésio: Na parte aérea, os tratamentos com solução completa e com omissão de Mg foram considerados iguais em absorção de $\mathrm{Mg}$, além de serem os tratamentos com as maiores reduções na concentração de $\mathrm{Mg}$. No entanto, a omissão de $\mathrm{K}$ foi a que promoveu a maior concentração de $\mathrm{Mg}$, evidenciando o antagonismo existente entre esses macronutrientes. De acordo com Malavolta et al. (1997), a ausência de K na solução nutritiva aumenta a absorção de Mg pelas plantas, devido a uma inibição competitiva entre estes macronutrientes, os quais competem pelo mesmo sítio do carregador na absorção. A omissão de $\mathrm{N}$ provocou aumento da concentração de $\mathrm{Mg}$ na parte radicular das mudas, enquanto que nas omissões de $\mathrm{P}, \mathrm{K}, \mathrm{Ca}$ e $\mathrm{S}$, as reduções mais significativas.

Enxofre: As omissões de $\mathrm{P}$ e $\mathrm{Mg}$ promoveram aumento significativo da concentração de $\mathrm{S}$ na parte aérea das mudas de cerejeira. Silva et al. (2005) também observaram grandes teores de S na massa seca da parte aérea do umbuzeiro no tratamento com omissão de P. Porém as omissões de K e Ca foram as que mais reduziram a concentração de $\mathrm{S}$, quando comparado ao tratamento completo. Contrariamente ao que foi observado por Sgarbi et al. (1999), em que, ao se omitir K, observou-se aumento na concentração de $\mathrm{S}$ tanto nas folhas novas como nas velhas. Na parte radicular, o tratamento completo foi significativamente superior aos outros tratamentos.

\section{CONCLUSÃO}

- As mudas de Amburana acreana apresentaram sintomas de deficiência de macronutrientes condizentes com os descritos na literatura e são facilmente caracterizáveis.

- As omissões de macronutrientes não limitaram os crescimentos em altura e em diâmetro de colo das mudas de A. acreana nas condições do experimento.

- As omissões de $\mathrm{P}, \mathrm{S}$ e $\mathrm{N}$ foram as que mais limitaram a produção de massa seca na parte aérea, e as de $\mathrm{P}, \mathrm{S}$ e $\mathrm{K}$ nas raízes.

- Houve maiores teores de N, P e K na parte aérea e de N e Mg nas raízes.

\section{REFERÊNCIAS}

BARROSO, D. G.; FIGUEIREDO, F. A. M. M. A.; PEREIRA, R. C.; MENDONCCA, A. V. R.; SILVA, L. C. Diagnóstico de deficiências de macronutrientes em mudas de teca. Revista Årvore, Viçosa, v. 29, n. 5, p. $671-679,2005$. 
Bello, E. P. B. C. S.; AlbuQuerQue, M. C. F.; GUIMARÃES, S. C.; MENDONÇA, E. A. F. de. Germinação de sementes de Amburana acreana (Ducke) A. C. Sm. submetidas a diferentes condições de temperatura e de estresse hídrico. Revista Brasileira de Sementes, Pelotas, v. 30, n. 3, p. 16 - 24, 2008.

CARVALHO, P. E. R. Espécies florestais brasileiras: recomendações silviculturais, potencialidades e uso da madeira. Colombo: Embrapa; CNPF, 1994. 640 p.

DELL, B.; MALAJCZUK, N.; GROVE, T. S. Nutrient disorders in plantation eucalypts. Canberra: Australian Centre for International Agricultural Research, 1995. 104 p.

FONTES, P. C. R. Diagnóstico do estado nutricional das plantas. Viçosa: UFV, 2001. 122 p.

HUMMEL, A. C.; MINETTE L. J. Aspectos do setor florestal do estado da Amazônia. In: CONGRESSO FLORESTAL BRASILEIRO, 6., 1990, Campos do Jordão. Anais... São Paulo: SBS, 1990. p. 157 - 165.

KAUL, O. N.; SRIVASTAVA, P. B. L.; TANDON, V. N. Nutrition studies on Eucalyptus: 3 - diagnosis of mineral deficiences in Eucalyptus grandis seedlings. Indian forester, v. 94, n. 11, p. 831 - 834, 1968.

LIMA, M. M. Crescimento, composição mineral e sintomas de deficiências de macronutrientes em plantas de cupuaçuzeiro (Theobroma grandiflorum). 80 f. Dissertação (Mestrado em Agronomia) Universidade Federal Rural da Amazônia, Belém, 2002.

LOCATELli, M.; MACEDO, R. S.; VIEIRA, A. H. Caracterização de sintomas de deficiências em cedro-rosa (Cedrela odorata L.). Porto Velho: Embrapa Rondônia, 2006. 4 p. (Circular Técnica, n. 88).

MALAVOLTA, E. ABC da análise de solos e folhas. São Paulo: CERES, 1992. 124 p.

MAlavolta, E.; VITTI, G. C.; OLIVEIRA, S. A. de. Avaliação do estado nutricional das plantas: princípios e aplicações. 2 ed. Piracicaba: POTAFOS, 1997. 319 p.

MALÉZIEUX, E., BARTHOLOMEW, D. P. Plant Nutrition. In: BARTHOLOMEW, D. P.; PAUL, R. E., ROHRBACH, K. G. (Ed.). The pineapple - botany, production and uses. Honolulu: CABI Publishing, 2003. p. 143 - 165.

MAYNARD, D. N. The effects of nutrient stems on the growth and composition of spinach. Journal of the America Society for Horticultural Science, Alexandria, v. 95, n. 5, p. 598 - 600, 1970.

MARQUES, T. C. L. L. S. M.; CARVALHO, J. G. de.; LACERDA, M. P. C.; MOTA, P. E. F. da. Crescimento inicial do paricá (Schizolobium amazonicum) sob omissão de nutrientes e de sódio em solução nutritiva. Cerne, Lavras, v. 10, n. 2, p. 184 - 195, 2004.

MENDONÇA, A. V. R.; NOGUEIRA, F. D.; VENTURIN, N.; SOUZA, J. S. Exigências nutricionais de Myracrodruon urundeuva Fr. All (aroeira do sertão). Cerne, Lavras, v. 5, n. 2, p. 65 - 75, 1999.

RAMOS, M. J. M. Caracterização de sintomas de deficiência de macronutrientes e de boro em abacaxizeiro cultivar imperial. 95 p. Tese (Doutorado em Produção Vegetal) - Universidade Estadual do Norte Fluminense Darcy Ribeiro, Campos dos Goytacazes, 2006.

SARCINELLI, T. S.; RIBEIRO JR., E. S.; DIAS, L. E.; LYNCH, L. S. Sintomas de deficiência nutricional em mudas de Acacia holosericea em resposta à omissão de macronutrientes. Revista Árvore, Viçosa, v. 28, n. 2, p. 173 - 181, 2004.

SARRUGE, J. R. Soluções nutritivas. Summa Phytopathologica, Jaguariúna, v. 1, n. 3, p. 231 - 233 , 1975.

SGARBI, F; SILVEIRA, R. L. V. A.; TAKAHASHI, E. N.; CAMARGO, M. A. F. de. Crescimento e produção de biomassa de clone de Eucalyptus grandis x Eucalyptus urophylla em condições de deficiência de macronutrientes, B e Zn. Scientia Forestalis, Piracicaba, n. 56, p. 69 - 82, 1999.

SILVA, E. B.; GONÇALVES, N. P.; PINHO, P. J. de. Limitações nutricionais para crescimento de mudas de umbuzeiro em Latossolo Vermelho distrófico no Norte de Minas. Acta Scientiarum. Agronomy, Maringá, v. 27, n. 1, p. 55 - 59, 2005. 
SILVEIRA, R. L. V. A.; HIGASHI, E. N.; SGARBI, F.; MUNIZ, M. R. A. Informações Agronômicas, Piracicaba, n. 93, 2001.

SILVEIRA, R. L. V. A.; MOREIRA, A.; TAKASHI, E. N.; SGARBI, F.; BRANCO, E. F. Sintomas de deficiência de macronutrientes e de boro em clones híbridos de Eucalyptus grandis com Eucalyptus urophylla. Cerne, Lavras, v. 8, n. 2, p. 107 - 116, 2002.

SIMÕES, J. W.; COUTO, H. T. Z. Efeito da omissão de nutrientes na alimentação mineral do pinheiro do Paraná Araucaria Angustifolia (BERT.) O. Ktze. cultivado em vaso. IPEF, Piracicaba, n. 7, p. 3 - 39, 1973.

Veloso, C. A. C.; Muraoka, T.; Malavolta, E.; CARvalho, J. G. de. Diagnose de deficiências de macronutrientes em pimenteira-do-reino. 1998. Disponível em: <www.nutricaodeplantas.agr.br/site/downloads/.../omissao_pimenta2.doc?>. Acesso em: 29/06/2010.

WALlAU, R. de.; LUIZ, R.; BORGES, A. R.; REZENDE, A. R.; REZENDE, D. A.; CAMARGOS, S. L. Sintomas de deficiências nutricionais em mudas de mogno cultivadas em solução nutritiva. Cerne, Lavras, v. 14, n. 4, p. 304 - 310, 2008. 